\title{
Orbital optimized unitary coupled cluster theory for quantum computer
}

\author{
Wataru Mizukami $\odot,{ }^{1,2,3,{ }^{*}}$ Kosuke Mitarai, ${ }^{1,2,4, \dagger}$ Yuya O. Nakagawa, ${ }^{4}$ \\ Takahiro Yamamoto, ${ }^{4}$ Tennin Yan, ${ }^{4}$ and Yu-ya Ohnishi ${ }^{5, \ddagger}$ \\ ${ }^{1}$ Graduate School of Engineering Science, Osaka University, 1-3 Machikaneyama, Toyonaka, Osaka 560-8531, Japan \\ ${ }^{2}$ Center for Quantum Information and Quantum Biology, Institute for Open and Transdisciplinary Research Initiatives, \\ Osaka University, Osaka 560-8531, Japan \\ ${ }^{3}$ JST, PRESTO, 4-1-8 Honcho, Kawaguchi, Saitama 332-0012, Japan \\ ${ }^{4}$ QunaSys Inc., Aqua Hakusan Building 9F, 1-13-7 Hakusan, Bunkyo, Tokyo 113-0001, Japan \\ ${ }^{5}$ Materials Informatics Initiative, RD Technology \& Digital Transformation Center, JSR Corporation, 100 Kawajiri-cho, \\ Yokkaichi, Mie, 510-8552, Japan
}

(Received 23 March 2020; accepted 11 August 2020; published 16 September 2020)

\begin{abstract}
We propose an orbital optimized method for unitary coupled cluster theory (OO-UCC) within the variational quantum eigensolver (VQE) framework for quantum computers. OO-UCC variationally determines the coupled cluster amplitudes and also molecular orbital coefficients. Owing to its fully variational nature, first-order properties are readily available. This feature allows the optimization of molecular structures in VQE without solving any additional equations. Furthermore, the method requires smaller active space and shallower quantum circuits than UCC to achieve the same accuracy. We present numerical examples of OO-UCC using quantum simulators, which include the geometry optimization of water and ammonia molecules using analytical first derivatives of the VQE.
\end{abstract}

DOI: 10.1103/PhysRevResearch.2.033421

\section{INTRODUCTION}

Coupled cluster theory $(\mathrm{CC})$ is one of the most representative electron correlation methods in quantum chemistry [1-3]. It has some vital features to describe molecular electronic structures reliably. CC is size-extensive and can be improved systematically by increasing the excitation level. It converges to full configuration interactions (FCI) faster than truncated configuration interactions (CI) or Møller-Plesset perturbation theory. Furthermore, its energy is invariant to unitary transformations among the occupied/virtual orbitals. It is well known that for an electronic state where the mean-field approximation works well, the $\mathrm{CC}$ models considering up to triple excitations can provide chemical accuracy (e.g., $1 \mathrm{kcal} / \mathrm{mol}$ ), if sufficiently large basis functions are employed. In particular, it has been established that CC singles and doubles with perturbative triples (i.e., CCSD (T)), which incorporates three-body interactions perturbatively, is a highly accurate method. It is often called the gold standard of molecular electronic structure theory [4].

In the framework of the traditional CC (TCC), the wavefunction parameters are determined by solving projected amplitude equations and not variationally. Owing to its nonva-

\footnotetext{
*wataru.mizukami.857@qiqb.otri.osaka-u.ac.jp

†mitarai@qc.ee.es.osaka-u.ac.jp

†Yuuya_Oonishi@jsr.co.jp
}

Published by the American Physical Society under the terms of the Creative Commons Attribution 4.0 International license. Further distribution of this work must maintain attribution to the author(s) and the published article's title, journal citation, and DOI. riational properties, the validity of TCC is strongly dependent on the reference Hartree-Fock wave function. Indeed, CCSD and $\operatorname{CCSD}(\mathrm{T})$ often suffer from breakdowns in the systems where static electron correlations are strong, for example, multiple-chemical-bond breaking systems [1].

This issue can be solved if a CC wave function is parametrized variationally [5]. Some benchmark studies showed that the difference between the variational CC (VCC) and TCC is small for weakly correlated regions [5-7]. Nonetheless, VCC has a factorial scaling in the computational cost. Therefore it is only applicable to a tiny system where FCI can be performed. However, it has recently been shown that a variant of VCC - unitary coupled cluster (UCC) [8-15]can be solved at a polynomial-scaling cost using a quantum computer [16]. A UCC wave function can be prepared on a quantum computer using the Trotter approximation with a polynomial number of quantum gates. Although the gate count for the accurate UCC can be much larger than what today's quantum devices are capable of [17], UCC can be a good starting point for analyzing the power of the quantum computer in the field of quantum chemistry.

The method enabling UCC on a quantum computer is called variational quantum eigensolver (VQE), which is a kind of quantum-classical hybrid algorithm [16]. In the $\mathrm{VQE}$, a wave function is prepared through a parametrized quantum circuit corresponding to a wave function ansatz (e.g., UCC). Then, we measure its energy for given circuit parameters. The parameters of the circuit are iteratively tuned by a nonlinear optimizer running on a classical computer to minimize the energy. VQE calculations using actual quantum computers have already been performed for small molecules [16,18-23]. Recently, researchers have proposed 
electron-correlation methods based on UCC for quantum computers [24-33].

Although VQE allows the determination of UCC parameters based on the Rayleigh-Ritz variational procedure, the obtained UCC wave functions are not fully variational. UCC and its variants employ a Hartree-Fock determinant as a reference wave function; the orbitals are fixed and not altered during a UCC calculation. However, it is well known that the Hartree-Fock orbitals are not optimal orbitals for a correlated wave function. One method for obtaining such optimal orbitals is to optimize orbitals in such a way that the gradients of the energy with respect to orbital rotation parameters vanish. The combination of CC with orbital optimization (OO-CC) was first briefly mentioned in the paper of Purvis and Bartlett and then introduced by Sherrill et al. [34,35]. Since then, orbital optimized coupled cluster doubles (OO-CCD) and its variants have been developed by various researchers [35-52].

This paper concerns the orbital-optimization technique to UCC in the context of VQE. At this moment, the size of the orbital space that can be handled by a quantum computer is severely limited because of the number of available qubits. Therefore active space approximation is indispensable when we wish to use a quantum computer for quantum chemical problems. Improvement of the active space can be achieved by optimizing molecular orbitals with the VQE, which leads to a reduced number of qubits. Furthermore, the orbital-optimized $\mathrm{VQE}(\mathrm{OO}-\mathrm{VQE})$ is a fully variational method, and the molecular gradients of OO-VQE (e.g., forces) can be calculated without solving response equations. The idea of using the orbital-optimization techniques for quantum computers has already been reported by Reiher $\mathrm{et}$ al. for the phase estimation algorithm (PEA) [53] and by Takeshita et al. for the VQE [54].

In this study, we implement OO-VQE using a quantum circuit simulator; we propose an orbital optimized unitary coupled cluster doubles (OO-UCCD) as a wave-function model for OO-VQE. It must be noted that after posting the initial manuscript of this work on the arXiv preprint server, an implementation of OO-UCCD by Sokolov et al. was uploaded on arXiv [55]. Their study carefully performed the cost and accuracy analysis of several variants of OO-UCCD methods, while our work focused on its fully variational nature and computed analytical derivatives for geometry optimizations which are a vital part of quantum chemical calculations. To the best of our knowledge, this is the first time that geometry optimizations have been performed for polyatomic molecules using analytic first derivatives of the VQE and ab initio Hamiltonian.

The remainder of this paper is organized as follows. First, Sec. II describes the theory of orbital optimized UCC (OOUCC) based on VQE. Section III provides a brief description of the implementation of OO-UCC using a quantum circuit simulator. Section III also discusses simple numerical experiments to demonstrate its usefulness. Finally, Sec. IV concludes the paper.

\section{THEORY}

\section{A. Unitary coupled cluster}

The molecular electronic Hamiltonian in a spin-free form is expressed as

$$
\hat{H}=\sum_{p, q} h_{p q} \hat{E}_{p q}+\sum_{p, q, r, s} h_{p q r s}\left\{\hat{E}_{p q} \hat{E}_{r s}-\delta_{q r} \hat{E}_{p s}\right\},
$$

where $h_{p q}$ and $h_{p q r s}$ are one- and two-electron integrals, respectively. $\hat{E}_{p q}$ is a singlet excitation operator and is defined as $\hat{E}_{p q}=\hat{c}_{p, \alpha}^{\dagger} \hat{c}_{q, \alpha}+\hat{c}_{p, \beta}^{\dagger} \hat{c}_{q, \beta}$, where $\hat{c}_{p, \alpha}^{\dagger}$ and $\hat{c}_{p, \beta}$ are creation and annihilation second quantized operators, respectively. $p, q, r, s$ are the indices of general molecular spatial orbitals.

A wave function in the traditional coupled cluster ansatz is given as

$$
|\Psi\rangle=e^{\hat{T}}|0\rangle,
$$

where $\hat{T}$ is an excitation operator $\hat{T}=\hat{T}_{1}+\hat{T}_{2}+\hat{T}_{3}+\cdots$ and $|0\rangle$ is a reference wave function. In contrast, UCC uses an anti-Hermite operator $\hat{A}$ defined by the difference of the amplitude operator $\hat{T}$ of TCC and its Hermitian conjugate, i.e., $\hat{A}=\hat{T}-\hat{T}^{\dagger}$. Therefore a wave function of the UCC ansatz is expressed as

$$
|\Psi\rangle=e^{\hat{A}}|0\rangle .
$$

The Baker-Campbell-Hausdorff (BCH) expansion of the similarity transformed Hamiltonian of the traditional $\mathrm{CC}$ is terminated at the finite order, whereas that of UCC is not, owing to de-excitation operators $\hat{T}^{\dagger}$. The infinite $\mathrm{BCH}$ expansion makes the implementation of UCC on a classical computer unfeasible.

\section{B. Orbital optimization}

Optimizing orbitals is equivalent to minimizing a wave function with respect to orbital rotation parameters $\kappa$. The energy function of OO-UCC is given by

$$
E(A, \kappa)=\left\langle\Psi\left|e^{-\hat{\kappa}} \hat{H} e^{\hat{\kappa}}\right| \Psi\right\rangle=\left\langle 0\left|e^{-\hat{A}} e^{-\hat{\kappa}} \hat{H} e^{\hat{\kappa}} e^{\hat{A}}\right| 0\right\rangle,
$$

where the orbital rotation operator is defined as $\hat{\kappa}=$ $\sum_{p q} \kappa_{p q}\left(\hat{E}_{p q}-\hat{E}_{q p}\right)$. When UCC parameters $A$ are fixed, the second order expansion of the energy function becomes

$$
\begin{aligned}
E(A, \kappa) \approx & \langle\Psi|\hat{H}| \Psi\rangle+\sum_{p q} \kappa_{p q}\left\langle\Psi\left|\left[\hat{H}, \hat{E}_{p q}^{-}\right]\right| \Psi\right\rangle \\
& +\frac{1}{2} \sum_{p q, r s} \kappa_{p q}\langle\Psi|\left[\left[\hat{H}, \hat{E}_{p q}^{-}\right], \hat{E}_{r s}^{-}\right] \\
& +\left[\left[\hat{H}, \hat{E}_{r s}^{-}\right], \hat{E}_{p q}^{-}\right]|\Psi\rangle \kappa_{r s},
\end{aligned}
$$

where $\hat{E}_{p q}^{-}=\hat{E}_{p q}-\hat{E}_{q p}$. By taking the derivative with respect to $\kappa$, the following Newton-Raphson equation is obtained

$$
\mathbf{H} \kappa=-\mathbf{g},
$$

whose elements are

$$
\begin{gathered}
H_{p q, r s}=\frac{1}{2}\left\langle\Psi\left|\left[\left[\hat{H}, \hat{E}_{p q}^{-}\right], E_{r s}^{-}\right]+\left[\left[\hat{H}, \hat{E}_{r s}^{-}\right], \hat{E}_{p q}^{-}\right]\right| \Psi\right\rangle \\
g_{p q}=\left\langle\Psi\left|\left[\hat{H}, \hat{E}_{p q}^{-}\right]\right| \Psi\right\rangle .
\end{gathered}
$$

$H$ and $g$ are often called electronic Hessian and gradients, respectively. One-particle and two-particle reduced density matrices (1RDM and 2RDM) are required to compute them in addition to the molecular Hamiltonian integrals $h_{p q}$ and $h_{\text {pqrs }}$. They are readily available in VQE, because it measures $1 \mathrm{RDM}$ and $2 \mathrm{RDM}$ to compute electronic energy in a given quantum circuit. 


\section{Orbital optimized unitary coupled cluster doubles}

The UCC singles and doubles (UCCSD) can be expressed as

$$
\left|\Psi^{\mathrm{UCCSD}}\right\rangle=e^{\hat{A}_{1}+\hat{A}_{2}}|0\rangle,
$$

where $\hat{A}_{n}=\hat{T}_{n}-\hat{T}_{n}^{\dagger}$ consists of $n$-excitation operators $\hat{T}_{n}$ and their conjugates. Starting from the UCCSD ansatz (9), we consider the following wave-function model by separating the singles and doubles parts:

$$
\left|\Psi^{\mathrm{UCCSD}^{\prime}}\right\rangle=e^{\hat{A}_{2}} e^{\hat{A}_{1}}|0\rangle .
$$

The UCC operator $\hat{A}$ is not commutable unlike TCC, because of the existence of de-excitation operators $\hat{T}^{\dagger}$. Therefore the decomposed UCCSD ansatz is different from the original ansatz. The singles part $e^{\hat{A}_{1}}$ in this model is identical to the orbital rotation unitary operator $e^{\hat{\kappa}}$ that is present in Eq. (4). This implies that we can optimize its singles part $e^{\hat{A}_{1}}$ variationally using a classical computer via the wellestablished orbital-optimization technique. The singles only alter the Hartree-Fock determinant to another determinant $|\tilde{0}\rangle=e^{\hat{A}_{1}}|0\rangle$.

Considering the Slater determinant $|\tilde{0}\rangle$ as a reference wave function for UCC, we rewrite Eq. (10) and propose the orbital-optimized unitary coupled cluster doubles (OOUCCD) model, given as

$$
\left|\Psi^{\mathrm{OO}-\mathrm{UCCD}}\right\rangle=e^{\hat{\tilde{A}}_{2}}|\tilde{0}\rangle .
$$

The doubles part $e^{\hat{A}_{2}}$ in Eq. (11) is optimized by the VQE, while the reference determinant (i.e., the singles) is optimized by a classical computer using 1RDM and 2RDM from VQE. In the framework of VQE, these quantities need to be evaluated via measurements. Formally, measuring 2RDM scales to the fourth power with respect to the number of qubits, and it is a major bottleneck not only in VQE but also in OO-VQE. In addition, the measurement introduces statistical errors. In that sense, OO-UCCD (OO-VQE) is related to the multiconfigurational self-consistent field methods; these are based on stochastic electron correlation approaches such as full configuration interaction quantum Monte Carlo [56,57], the heat-bath CI [58], and variational Monte Carlo [59-64]. The costs and errors owing to the measurements can be reduced using simultaneous measurements and/or other techniques. Such techniques have been actively developed [65-75], and they can be used to alleviate the problems caused by measuring 1 and 2RDMs.

In practice, OO-VQE repeatedly performs the VQE and the orbital optimization until convergence. The computational cost of OO-UCCD is, therefore, higher than that of UCCSD (or UCCD) by a factor of the number of iterations (typically several to several tens of times). Nonetheless, since the number of iterations is independent of the size of a molecule, OO-UCCD is applicable to the problems where UCCD (or UCCSD) is feasible in terms of computational time. In return for this increase in computational cost owing to self-consistency, Eq. (11) has the advantage that all the wave function parameters are fully variationally determined, and it requires a less complicated quantum circuit than Eq. (10). This property makes it easy to compute the first derivative and simplifies the higher-order derivative computations, as discussed in the next subsection. Furthermore, OO-UCCD can also incorporate correlations outside the active space. Similar to other existing orbital optimization methods, this feature is beneficial, especially for the system where the orbital relaxation effects play crucial roles, and the number of orbitals is larger than the number of available qubits (e.g., transitionmetal complexes).

\section{Analytical first derivatives of energy}

An important advantage of OO-VQE including OO-UCCD is that all the wave-function parameters are variationally determined. This feature allows us to compute first analytical derivatives of the energy without solving any additional equation. They are vital quantities for quantum chemical calculations, because static molecular properties such as forces on nuclei are defined as the derivatives of the energy with respect to external parameters $x$ (such as a position of atom or an external electric field). Here, the energy is considered as a function of external parameters $x$, the VQE circuit parameters $\theta$ and the orbital parameters $\kappa$, denoted as $E(x, \theta, \kappa)$ in this subsection. Then, the first derivatives of the VQE energy are given as

$$
\begin{aligned}
\frac{d E(x, \theta, \kappa)}{d x}= & \frac{\partial E(x, \theta, \kappa)}{\partial x}+\frac{\partial E(x, \theta, \kappa)}{\partial \theta} \frac{\partial \theta}{\partial x} \\
& +\frac{\partial E(x, \theta, \kappa)}{\partial \kappa} \frac{\partial \kappa}{\partial x},
\end{aligned}
$$

where $\theta$ and $\kappa$ indicate the quantum circuit parameters and molecular orbital parameters, respectively. The first term of the right-hand side of Eq. (12) is the Hellmann-Feynman term and is equivalent to the expectation value of the derivative of the Hamiltonian. The second term is zero since the VQE variationally determines $\theta$. In the same way, the third term is also zero when the orbital is optimized. Thus the first-order OO-VQE energy derivatives can be determined by evaluating the expectation value of the derivative of the Hamiltonian.

This is not the case for the standard VQE without OO, which needs the third term to compute the first derivatives. To obtain the orbital response $\partial \kappa / \partial x$ in that term, we must solve the following first-order coupled-perturbed HartreeFock (CPHF) equation:

$$
\frac{\partial^{2} E_{\mathrm{HF}}(x, \kappa)}{\partial \kappa \partial \kappa} \frac{\partial \kappa}{\partial x}=\frac{\partial^{2} E_{\mathrm{HF}}(x, \kappa)}{\partial x \partial \kappa},
$$

where $E_{\mathrm{HF}}(x, \kappa)$ is the Hartree-Fock energy. In practice, we use the $\mathrm{Z}$-vector technique [76,77] so that we can avoid solving Eq. (13) for each of the external parameters (e.g. each Cartesian coordinate of a molecule) [78]. The second or higher-order derivatives require not only the orbital response but also the circuit parameter response. The latter can be computed analytically on a quantum computer using a method recently developed by a few of authors of this study [79].

\section{E. Trotterization and Brueckner orbitals}

Translating the UCC generator $e^{\hat{A}}$ into a quantum circuit needs Trotterization, which introduces an error owing to the finite Trotter number. Hereafter, we denote UCCSD 
and UCC doubles (UCCD) approximated by the $n$-step Trotter expansion as $\mathrm{UCCSD}_{n}$ and $\mathrm{UCCD}_{n}$, respectively. The wave-function ansatz, which we implement in this study, corresponds to OO-UCCD ${ }_{1}$, where the Trotter expansion is truncated at the very first step. The OO-UCCD ${ }_{1}$ ansatz can be written as

$$
\begin{aligned}
|\Psi\rangle & =\prod_{\mu}\left(e^{\hat{A}_{2, \mu}}\right) e^{\hat{A}_{1}}|0\rangle \\
& =\prod_{\mu}\left(e^{\hat{\hat{A}}_{2, \mu}}\right)|\tilde{0}\rangle,
\end{aligned}
$$

where $\mu$ is an index for each double excitation (and de-excitation) operator and $\hat{A}_{2}=\sum_{\mu} \hat{A}_{2, \mu}$. Although a singleTrotter-step UCC ansatz appears to be a crude approximation, Barkoutsos et al. have shown that it actually reproduces ground-state energy accurately [80]. O'Malley et al. have pointed out that the variational flexibility allows such an approximated wave function model to absorb the Trotterization error [18].

Note that the Trotter error depends on the ordering of the operators $[81,82]$. This order dependency makes $\mathrm{UCCSD}_{n}$ inequivalent to OO-UCCD ${ }_{n}$ even when $n=1$. The orbital rotations of OO-UCCD 1 alter the doubles $\hat{A}_{2}$ in HartreeFock orbitals to $\hat{\tilde{A}}_{2}$ in OO-UCCD 's optimized orbitals, which appear in Eqs. (11) and (14). Because of this change, the ordering of the doubles in OO-UCCD 1 is not matched to that in $\mathrm{UCCSD}_{1}$. There is, thus, a difference between $\mathrm{UCCSD}_{1}$ and OO-UCCD 1 caused by the dependency on the ordering of the doubles. Izmaylov et al. have recently proposed a way to reduce the order dependency based on the Lie algebra-Lie group connection [82].

A notable feature of the OO-UCCD ansatz is that the variational orbitals coincide with the commonly known Brueckner orbitals. Brueckner orbitals are optimal orbitals for a correlated wave function, where the singles' contribution (i.e., $\hat{T}_{1}$ or $\hat{A}_{1}$ ) vanishes. It is known that in the TCC framework, the variationally optimized orbitals are not the same as the Brueckner orbitals. This is because of the difference between $\hat{T}_{1}$ and the orbital rotation operators. Meanwhile, the singles of UCC $\hat{A}_{1}$ are identical to the orbital rotatioon operators. Nonetheless, the Brueckner orbitals of UCCSD are not identical to the OO-UCCSD optimzied orbitals because the anti-Hermitian operators $\hat{A}_{1}$ and $\hat{A}_{2}$ are not generally commutable with each other and the Trotterization makes a difference. The OOUCCD ansatz is based on the separation of the singles and doubles a posteriori as shown in Eq. (10). Therefore the OOUCCD naturally satisfies the Brueckner condition $e^{\hat{A}_{1}}=0$ and the variational condition $\frac{\partial E}{\partial \kappa}=0$, simultaneously: variational orbitals are Brueckner orbitals in this ansatz.

\section{NUMERICAL EXAMPLES}

In this section, we present some numerical results using the proposed method. Computational details are as follows. We have implemented OO-UCCD in Python using Qulacs, PySCF [83], and OpenFermion [84] program packages. Qulacs is used to simulate quantum circuits. PYSCF is used for orbital optimizations and for evaluating molecular Hamiltonian integrals [85]. OpenFermion is employed for mapping molecular Hamiltonian into a quantum circuit based on the Jordan-Wigner transformation. 1 and 2RDMs are computed not by sampling but by directly using a state vector. This implies that we have not considered (statistical) noise.

We employ the following ordering of the Trotterized operators on the quantum circuits for $\mathrm{UCCSD}_{1}\left(\mathrm{UCCD}_{1}\right)$ : first, the doubles from two spatial occupied-virtual pairs; second, the doubles from the same spatial occupied-virtual pairs; finally, the singles. The double excitation operators $\hat{T}_{n}=t_{p q}^{r s} \hat{c}_{p}^{\dagger} \hat{c}_{q}^{\dagger} \hat{c}_{r} \hat{c}_{s}$ are generated so that the index strings psqr are in lexicographical order, where $t_{p q}^{r s}$ is a doubles amplitude. Although Grimsley et al. have illustrated that the effect of the operator ordering could be significant, their results suggest that the impact of the ordering is not important in the systems used in this study [81].

\section{A. Without the active space approximation}

In this section, we show the results without the active space approximation. It means that all the orbitals were mapped into qubits. Then, the results with active space approximation will be presented in the next subsection. Hereafter, we denote the OO-UCCD and UCCSD using the active space approach as S-OO-UCCD and AS-UCCSD, respectively, to distinguish them from no-active-space calculations. In addition, the active space sizes are explicitly written after the name of an electron correlation method whenever the approximation is used. Let OO-UCCD and MP2 with the active space consisting of 6 spatial orbitals and 4 electrons be written as $\mathrm{AS}-\mathrm{OO}-\mathrm{UCCD}(6 o, 4 e)$ and MP2 $(6 o, 4 e)$, respectively.

We first investigate the potential energy curve (PEC) of $\mathrm{LiH}$. Figure 1 shows the errors of our method with respect to the PECs computed by FCI and $\mathrm{UCCSD}_{1}$ with the STO-3G basis sets. We did not employ the active space approximation. It can be seen that the energy difference between $\mathrm{UCCSD}_{1}$ and OO-UCCD ${ }_{1}$ is notably small in the entire range of the PEC. The energy deviation of OO-UCCD from $^{U_{C C S D}}$ is $_{10} 0^{-7}$ mhartree at $1.3 \AA \mathrm{Li}-\mathrm{H}$ distance, whereas at $2.1 \AA$ it is $3 \times$ $10^{-5}$ mhatree. As mentioned above, the difference between $\mathrm{UCCSD}_{1}$ and $\mathrm{OO}-\mathrm{UCCD}_{1}$ essentially comes from the order dependency of the doubles. Indeed, Grimsley et al. reported that the typical doubles-operator-order dependency results in an energy difference of an order of less than a microhartree for $\mathrm{UCCSD}_{1}$ near the equilibrium geometry for $\mathrm{LiH}$, whereas the magnitude of the order-dependency increases with an increase in the $\mathrm{LiH}$ bond length [81]. Our results are consistent with theirs.

In contrast, the deviation of $\mathrm{UCCD}_{1}$ is at least five orders of magnitude larger than OO-UCCD ${ }_{1}$. OO-UCCD is as accurate as UCCSD; likewise the standard OO-CCD calculations reproduce the CCSD results well. This indicates that UCCSDlevel results can be obtained with a shallower quantum circuit using the orbital optimization technique at the cost of repeated VQE optimizations.

We next consider the double dissociation of the $\mathrm{H}_{2} \mathrm{O}$ molecule using the STO-3G basis sets, where the two $\mathrm{O}-\mathrm{H}$ bonds symmetrically stretch with a fixed $\mathrm{HOH}$ angle of $104.5^{\circ}$. Figure 2 illustrates that all the three unitary coupled cluster variants correctly describe the PEC of this reaction even in the bond-dissociation regime where four electrons are 
(a)

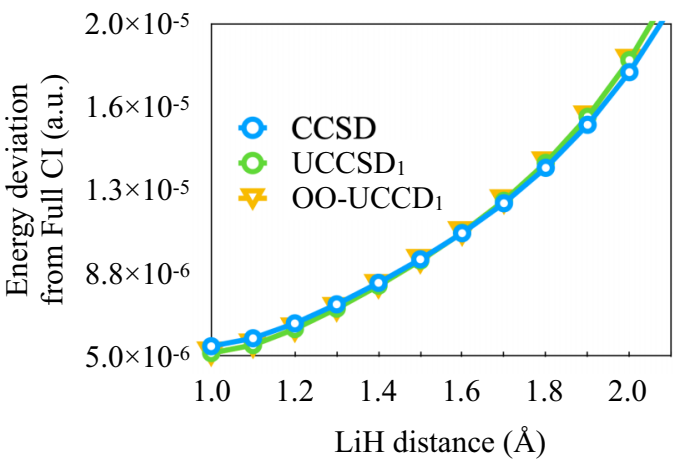

(b)

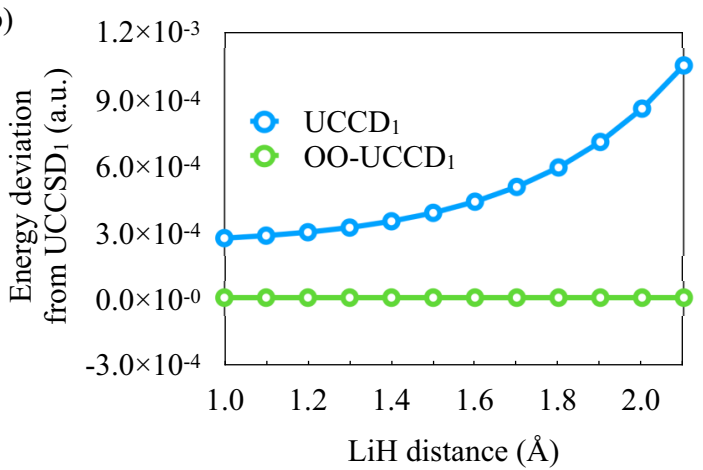

FIG. 1. Error of the OO-UCCD 1 method along with potential energy curves of the $\mathrm{LiH}$ molecule using the STO-3G basis sets. (a) Deviations from FCI energies compared with $\mathrm{UCCSD}_{1}$ and the standard CCSD method. (b) Deviations from $\mathrm{UCCSD}_{1}$ energies compared with $\mathrm{UCCD}_{1}$.

strongly correlated. Meanwhile, as is well known, the error of CCSD with respect to FCI increases with the length of the $\mathrm{O}-\mathrm{H}$ bonds, resulting in an artificial maximum at around $2.0 \AA$ [5]. Though the PECs of $\mathrm{UCCSD}_{1}$ and OO-UCCD behaves similar to FCI, their errors also increase as the $\mathrm{O}-\mathrm{H}$ distance increases. The deviations of $\mathrm{UCCSD}_{1}$ and OO$\mathrm{UCCD}_{1}$ from the FCI are within $1 \mathrm{kcal} / \mathrm{mol}$ if the $\mathrm{O}-\mathrm{H}$ bond length is less than $2 \AA$, while the error at $3 \AA$, where the PEC is almost flat, reaches $4 \mathrm{kcal} / \mathrm{mol}$. This behavior of UCC is close to the results of the variational coupled cluster theory reported by Van Voorhis and Head-Gordon [5].

Third, we report the geometry optimization of the water and ammonia molecules with STO-3G basis sets using the analytical derivatives of $\mathrm{UCCD}_{1}, \mathrm{UCCSD}_{1}$, and OO-UCCD $\mathrm{UCD}_{1}$. For comparison, we carried out Hartree-Fock, MP2, CCSD, CCSD(T), and FCI calculations. The CCSD and CCSD(T) geometries were obtained by the ORCA program package using numerical gradients [86,87]. All electrons were correlated in these calculations.

Table I shows the error of the total energy from FCI for each optimized geometry and the root mean square deviation (RMSD) error of each optimized structure. The RMSD error is a commonly used measure of the structural difference of two molecules. It was computed using the deviations of Cartesian coordinates of atoms from the reference FCI values, where the molecular structure was superimposed on the reference structure. It shows that $\mathrm{OO}-\mathrm{UCCD}_{1}$ is close to FCI
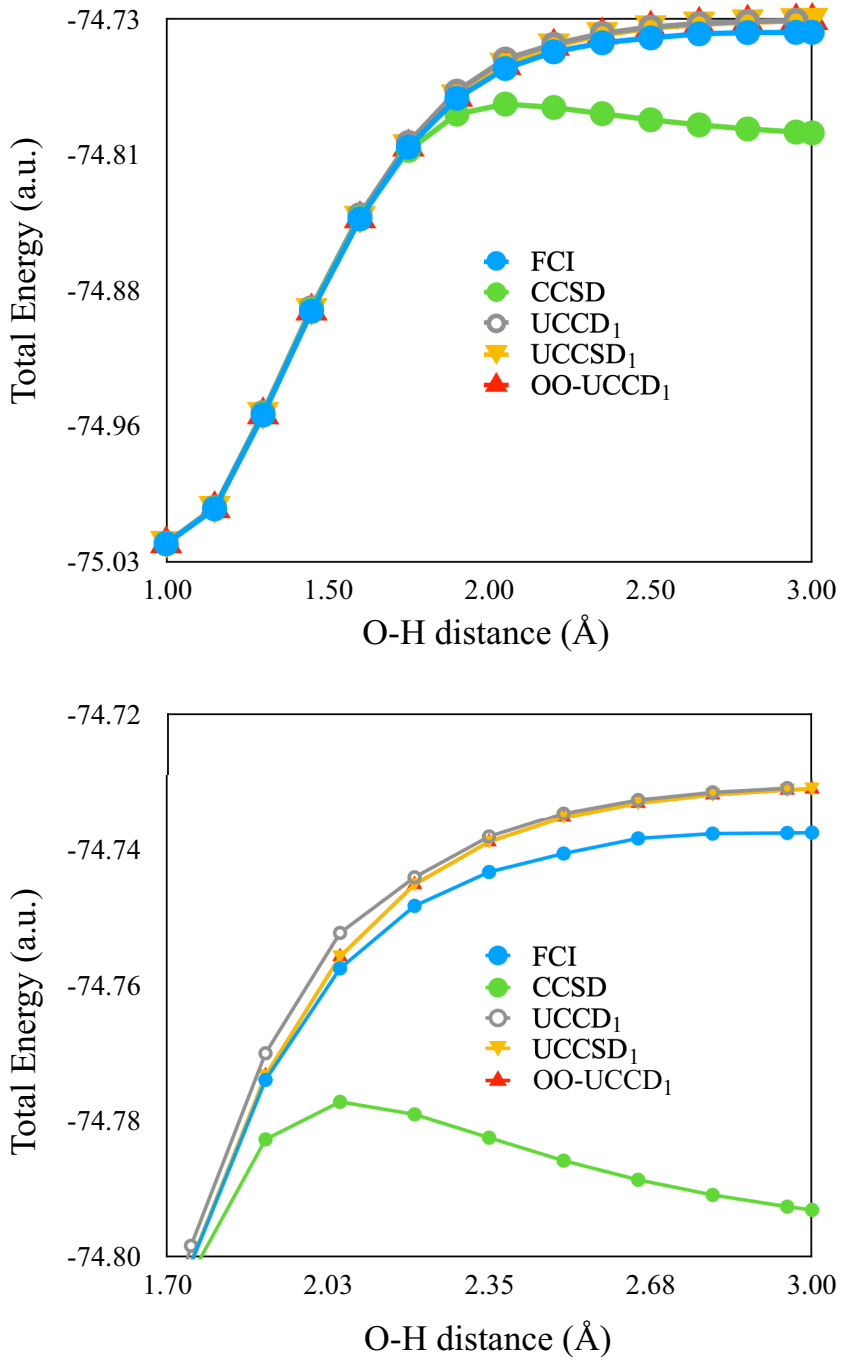

FIG. 2. Potential energy curves of the double dissociation of the water molecule's $\mathrm{OH}$ bonds computed at $\mathrm{OO}-\mathrm{UCCD}_{1}, \mathrm{UCCD}_{1}$, $\mathrm{UCCSD}_{1}, \mathrm{CCSD}$, and FCI using the STO-3G basis sets.

in terms of both structure (i.e., $3 \times 10^{-4} \AA$ ) and energy (i.e., 0.2 mhartree). One can see that $\mathrm{OO}-\mathrm{UCCD}_{1}$ and $\mathrm{UCCSD}_{1}$ provided virtually the same geometries and energies for these two systems, though they are not exactly same to each other

TABLE I. RMSD errors (in angstrom) for geometries optimized by OO-UCCD $/$ STO-3G and standard wave-function models HF, MP2, CCSD, and CCSD(T) relative to FCI. The parentheses show deviations of energies at optimized geometries from FCI.

\begin{tabular}{|c|c|c|}
\hline & $\mathrm{NH}_{3}$ & $\mathrm{H}_{2} \mathrm{O}$ \\
\hline $\mathrm{HF}$ & $3.2 \times 10^{-2}\left(7.3 \times 10^{-2}\right)$ & $2.4 \times 10^{-2}\left(5.7 \times 10^{-2}\right)$ \\
\hline MP2 & $9.0 \times 10^{-3}\left(2.1 \times 10^{-2}\right)$ & $9.3 \times 10^{-3}\left(1.7 \times 10^{-2}\right)$ \\
\hline CCSD & $2.9 \times 10^{-4}\left(2.5 \times 10^{-4}\right)$ & $1.7 \times 10^{-4}\left(1.5 \times 10^{-4}\right)$ \\
\hline $\mathrm{UCCD}_{1}$ & $5.1 \times 10^{-4}\left(3.7 \times 10^{-4}\right)$ & $5.1 \times 10^{-4}\left(4.1 \times 10^{-4}\right)$ \\
\hline $\mathrm{UCCSD}_{1}$ & $3.3 \times 10^{-4}\left(1.8 \times 10^{-4}\right)$ & $7.1 \times 10^{-5}\left(1.0 \times 10^{-4}\right)$ \\
\hline OO-UCCD 1 & $3.3 \times 10^{-4}\left(1.8 \times 10^{-4}\right)$ & $6.7 \times 10^{-5}\left(1.0 \times 10^{-4}\right)$ \\
\hline $\operatorname{CCSD}(\mathrm{T})$ & $8.1 \times 10^{-5}\left(1.3 \times 10^{-4}\right)$ & $1.7 \times 10^{-4}\left(7.8 \times 10^{-5}\right)$ \\
\hline
\end{tabular}




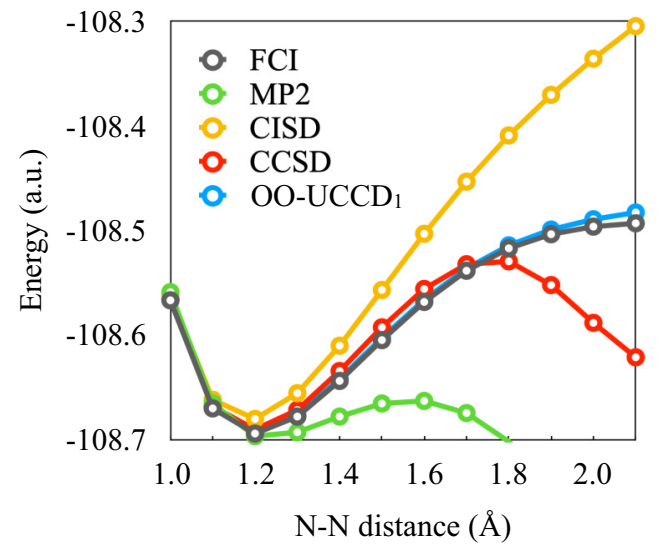

FIG. 3. Potential energy curves of the $\mathrm{N}_{2}$ molecule computed at $\mathrm{AS}-\mathrm{OOO}-\mathrm{UCCD}_{1}(6 o, 6 e), \operatorname{MP} 2(6 o, 6 e), \operatorname{CISD}(6 o, 6 e)$, $\operatorname{CCSD}(6 o, 6 e)$, and $\operatorname{FCI}(6 o, 6 e)$ using the STO-3G basis sets, where six orbitals and six electrons were correlated. Other orbitals were kept fixed and not used during all the post-Hartree-Fock calculations; here, the external orbital rotation was omitted for AS-OO-UCCD $1(6 o, 6 e)$.

owing to the order dependency of the doubles as explained above (e.g., $4 \mu \AA$ in the RMSD error of $\mathrm{H}_{2} \mathrm{O}$ ).

On the other hand, $\mathrm{UCCD}_{1}$ is less accurate than CCSD and the other two UCC models because of the lack of the singles contributions, though $\mathrm{UCCD}_{1}$ is more precise than MP2. The number of the VQE parameters $\theta$ and the depth of the quantum circuits of OO-UCCD $\mathrm{O}_{1}$ and $\mathrm{UCCD}_{1}$ were 120 and 2720, respectively, for $\mathrm{NH}_{3}$, while those of $\mathrm{UCCSD}_{1}$ were 135 and 2780 , respectively. These results demonstrate that the orbital-optimization allows us to slightly reduce the circuit depth while retaining the accuracy.

Furthermore, a comparison with CCSD and CCSD(T) suggests that $\mathrm{OO}-\mathrm{UCCD}_{1}$ 's energy is more accurate than that of CCSD and less accurate than that of $\operatorname{CCSD}(\mathrm{T})$ when electron correlation is weak. This tendency is consistent with the findings of Kühn et al. for the total energy and reaction energy [17]; in terms of geometry, OO-UCCD ${ }_{1}$ is slightly better than both CCSD and CCSD(T) for $\mathrm{H}_{2} \mathrm{O}$, while for $\mathrm{NH}_{3}$ it is close to CCSD and worse than $\operatorname{CCSD}(\mathrm{T})$.

\section{B. With the active space approximation}

Next, we examine the potential energy curve (PEC) of $\mathrm{N}_{2}$ using the active space approximation. This system, involving triple bond breaking, is a well-known benchmark for electron correlation methods, where the standard methods of many body perturbation theory such as MP2, CCSD, and $\operatorname{CCSD}(\mathrm{T})$ breakdown. We have computed the PEC at the AS-OO-UCCD $1(6 o, 6 e) / \mathrm{STO}-3 \mathrm{G}$ level of theory by fixing the lowest four occupied orbitals and eight electrons. Figure 3 shows the PEC along with those computed by MP2 $(6 o, 6 e)$, $\operatorname{CISD}(6 o, 6 e), \operatorname{CCSD}(6 o, 6 e)$, and $\operatorname{FCI}(6 o, 6 e)$. It shows that AS-OO-UCCD can treat a multiple-bond-breaking system appropriately where electrons of the breaking chemical bond are strongly correlated. In this system, the differences among ASUCCD, AS-OO-UCCD, and AS-UCCSD were small. They are at most $0.2 \mathrm{kcal} / \mathrm{mol}$ owing to the little orbital relaxation

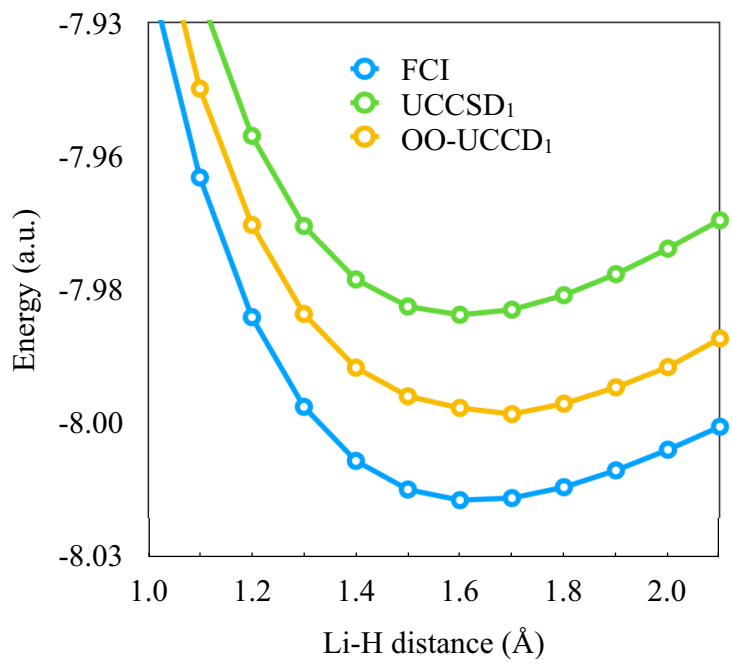

FIG. 4. Potential energy curves of the $\mathrm{LiH}$ molecule computed at $\mathrm{AS}-O O-\mathrm{UCCD}_{1}(4 o, 2 e), \mathrm{AS}-U C C S D_{1}(4 o, 2 e)$, and FCI using the 6-311G basis sets. AS-OO-UCCD ${ }_{1}$ and AS-UCCSD ${ }_{1}$ calculations employed active space consisting of four orbitals and two electrons [i.e., $(4 o, 2 e)]$, while all the orbitals and electrons were correlated for FCI.

effect in the small basis sets. The root mean square deviations (RMSD) of AS-UCCD $1(6 o, 6 e)$ and AS-OO-UCCD $1(6 o, 6 e)$ with respect to AS-UCCSD $(6 o, 6 e)$ PEC is $0.02 \mathrm{kcal} / \mathrm{mol}$ in the range of $1.0-2.1 \AA$.

The PEC of LiH with 6-311G basis sets, given in Fig. 4, is another example. For comparison, we computed the PEC by AS-OO-UCCD $1(4 o, 2 e)$ and also by AS-UCCSD $1(4 o, 2 e)$ and FCI. Only four orbitals and two electrons were correlated in these VQE calculations, and the orbital optimization was performed for all the orbitals and electrons. This example shows how the orbital optimization effectively considers the electron correlations outside the active space. Figure 4 illustrates that AS-OO-UCCD $1(4 o, 2 e)$ has lower energy and is closer to FCI without active space than AS-UCCSD $(4 o, 2 e)$, indicating that $\mathrm{AS}-\mathrm{OO}-\mathrm{UCCD}_{1}$ captures more electron correlation effects.

\section{SUMMARY}

In this work, we have developed OO-UCCD. OO-UCCD treats singles contributions not on quantum computers but on classical computers. All the wave function parameters are fully variationally determined in OO-UCC. This property makes the time-independent first-order properties readily available. Therefore geometry optimization or ab initio molecular dynamics can be performed using VQE without solving orbital response equations. Moreover, OO-UCCD incorporates the electron correlation effects outside the active space effectively. These aspects seem useful, especially in the age of noisy intermediate-scale quantum computers (NISQ) $[88,89]$, where the number of qubits and the coherence time are severely limited. Such an OO-VQE method may be useful for solving quantum chemical problems once quantum computers become commonplace. 


\section{ACKNOWLEDGMENTS}

This work was supported by MEXT Quantum Leap Flagship Program (MEXT Q-LEAP) Grants No. JPMXS0118067394 and No. JPMXS0118068682. W.M. wishes to thank JSPS KAKENHI No. 18K14181 and JST PRESTO No. JPMJPR191A. K.M. was supported by JSPS KAKENHI No. 19J10978. K.M. thanks for METI and IPA for their support through MITOU Target program. Some calculations were performed using the computational facilities in the Institute of Solid State Physics at the University of Tokyo, and in Research Institute for Information Technology (RIIT) at Kyushu University, Japan.

[1] R. J. Bartlett and M. Musiał, Rev. Mod. Phys. 79, 291 (2007).

[2] I. Shavitt and R. J. Bartlett, Many-Body Methods in Chemistry and Physics: MBPT and Coupled-Cluster Theory, Cambridge Molecular Science (Cambridge University Press, Cambridge, 2009).

[3] R. J. Bartlett, Wiley Interdiscip. Rev.: Comput. Mol. Sci. 2, 126 (2012).

[4] K. Raghavachari, G. W. Trucks, J. A. Pople, and M. HeadGordon, Chem. Phys. Lett. 157, 479 (1989).

[5] T. Van Voorhis and M. Head-Gordon, J. Chem. Phys. 113, 8873 (2000).

[6] B. Cooper and P. J. Knowles, J. Chem. Phys. 133, 234102 (2010).

[7] F. A. Evangelista, J. Chem. Phys. 134, 224102 (2011).

[8] W. Kutzelnigg, J. Chem. Phys. 77, 3081 (1982).

[9] W. Kutzelnigg and S. Koch, J. Chem. Phys. 79, 4315 (1983).

[10] W. Kutzelnigg, J. Chem. Phys. 82, 4166 (1985).

[11] R. J. Bartlett, S. A. Kucharski, and J. Noga, Chem. Phys. Lett. 155, 133 (1989).

[12] A. G. Taube and R. J. Bartlett, Int. J. Quantum Chem. 106, 3393 (2006).

[13] T. Yanai, Y. Kurashige, D. Ghosh, and G. K.-L. Chan, Int. J. Quantum Chem. 109, 2178 (2009).

[14] T. Yanai and G. K.-L. Chan, J. Chem. Phys. 124, 194106 (2006).

[15] G. Harsha, T. Shiozaki, and G. E. Scuseria, J. Chem. Phys. 148, 044107 (2018).

[16] A. Peruzzo, J. McClean, P. Shadbolt, M.-H. Yung, X.-Q. Zhou, P. J. Love, A. Aspuru-Guzik, and J. L. O’Brien, Nat. Commun. 5, 4213 (2014).

[17] M. Kühn, S. Zanker, P. Deglmann, M. Marthaler, and H. Weiß, J. Chem. Theory Comput. 15, 4764 (2019).

[18] P. J. J. O’Malley, R. Babbush, I. D. Kivlichan, J. Romero, J. R. McClean, R. Barends, J. Kelly, P. Roushan, A. Tranter, N. Ding, B. Campbell, Y. Chen, Z. Chen, B. Chiaro, A. Dunsworth, A. G. Fowler, E. Jeffrey, E. Lucero, A. Megrant, J. Y. Mutus, M. Neeley, C. Neill, C. Quintana, D. Sank, A. Vainsencher, J. Wenner, T. C. White, P. V. Coveney, P. J. Love, H. Neven, A. Aspuru-Guzik, and J. M. Martinis, Phys. Rev. X 6, 031007 (2016).

[19] A. Kandala, A. Mezzacapo, K. Temme, M. Takita, M. Brink, J. M. Chow, and J. M. Gambetta, Nature (London) 549, 242 (2017).

[20] C. Hempel, C. Maier, J. Romero, J. McClean, T. Monz, H. Shen, P. Jurcevic, B. P. Lanyon, P. Love, R. Babbush, A. AspuruGuzik, R. Blatt, and C. F. Roos, Phys. Rev. X 8, 031022 (2018).

[21] I. G. Ryabinkin, S. N. Genin, and A. F. Izmaylov, J. Chem. Theory Comput. 15, 249 (2018).

[22] A. J. McCaskey, Z. P. Parks, J. Jakowski, S. V. Moore, T. D. Morris, T. S. Humble, and R. C. Pooser, npj Quantum Inf. 5, 99 (2019).
[23] Q. Gao, H. Nakamura, T. P. Gujarati, G. O. Jones, J. E. Rice, S. P. Wood, M. Pistoia, J. M. Garcia, and N. Yamamoto, arXiv:1906.10675.

[24] I. G. Ryabinkin, T.-C. Yen, S. N. Genin, and A. F. Izmaylov, J. Chem. Theory Comput. 14, 6317 (2018).

[25] J. Lee, W. J. Huggins, M. Head-Gordon, and K. B. Whaley, J. Chem. Theory Comput. 15, 311 (2018).

[26] H. R. Grimsley, S. E. Economou, E. Barnes, and N. J. Mayhall, Nat. Commun. 10, 3007 (2019).

[27] W. J. Huggins, J. Lee, U. Baek, B. O'Gorman, and K. B. Whaley, New J. Phys 22, 073009 (2020).

[28] N. P. Bauman, E. J. Bylaska, S. Krishnamoorthy, G. H. Low, N. Wiebe, C. E. Granade, M. Roetteler, M. Troyer, and K. Kowalski, J. Chem. Phys. 151, 014107 (2019).

[29] N. P. Bauman, G. H. Low, and K. Kowalski, J. Chem. Phys. 151, 234114 (2019).

[30] I. G. Ryabinkin, R. A. Lang, S. N. Genin, and A. F. Izmaylov, J. Chem. Theory Comput. 16, 1055 (2020).

[31] Y. Matsuzawa and Y. Kurashige, J. Chem. Theory Comput. 16, 944 (2020).

[32] G. Greene-Diniz and D. M. Ramo, arXiv:1910.05168.

[33] F. A. Evangelista, G. K.-L. Chan, and G. E. Scuseria, J. Chem. Phys. 151, 244112 (2019).

[34] G. D. Purvis III and R. J. Bartlett, J. Chem. Phys. 76, 1910 (1982).

[35] C. D. Sherrill, A. I. Krylov, E. F. Byrd, and M. Head-Gordon, J. Chem. Phys. 109, 4171 (1998).

[36] A. Köhn and J. Olsen, J. Chem. Phys. 122, 084116 (2005).

[37] R. C. Lochan and M. Head-Gordon, J. Chem. Phys. 126, 164101 (2007).

[38] F. Neese, T. Schwabe, S. Kossmann, B. Schirmer, and S. Grimme, J. Chem. Theory Comput. 5, 3060 (2009).

[39] C. Kollmar and A. Heßelmann, Theor. Chem. Acc. 127, 311 (2010).

[40] U. Bozkaya, J. M. Turney, Y. Yamaguchi, H. F. Schaefer III, and C. D. Sherrill, J. Chem. Phys. 135, 104103 (2011).

[41] U. Bozkaya and H. F. Schaefer III, J. Chem. Phys. 136, 204114 (2012).

[42] J. B. Robinson and P. J. Knowles, J. Chem. Phys. 136, 054114 (2012).

[43] J. B. Robinson and P. J. Knowles, J. Chem. Phys. 138, 074104 (2013).

[44] T. N. Lan and T. Yanai, J. Chem. Phys. 138, 224108 (2013).

[45] U. Bozkaya and C. D. Sherrill, J. Chem. Phys. 139, 054104 (2013).

[46] K. Boguslawski, P. Tecmer, P. A. Limacher, P. A. Johnson, P. W. Ayers, P. Bultinck, S. De Baerdemacker, and D. Van Neck, J. Chem. Phys. 140, 214114 (2014).

[47] U. Bozkaya, Phys. Chem. Chem. Phys. 18, 11362 (2016). 
[48] D. Kats and D. P. Tew, J. Chem. Theory Comput. 15, 13 (2018).

[49] J. Lee and M. Head-Gordon, J. Chem. Theory Comput. 14, 5203 (2018).

[50] R. H. Myhre, J. Chem. Phys. 148, 094110 (2018).

[51] D. P. Tew and D. Kats, J. Chem. Theory Comput. 14, 5435 (2018).

[52] T. Sato, H. Pathak, Y. Orimo, and K. L. Ishikawa, J. Chem. Phys. 148, 051101 (2018).

[53] M. Reiher, N. Wiebe, K. M. Svore, D. Wecker, and M. Troyer, Proc. Natl. Acad. Sci. 114, 7555 (2017).

[54] T. Takeshita, N. C. Rubin, Z. Jiang, E. Lee, R. Babbush, and J. R. McClean, Phys. Rev. X 10, 011004 (2020).

[55] I. Sokolov, P. K. Barkoutsos, P. J. Ollitrault, D. Greenberg, J. Rice, M. Pistoia, and I. Tavernelli, J. Chem. Phys. 152, 124107 (2020).

[56] R. E. Thomas, Q. Sun, A. Alavi, and G. H. Booth, J. Chem. Theory Comput. 11, 5316 (2015).

[57] G. Li Manni, S. D. Smart, and A. Alavi, J. Chem. Theory Comput. 12, 1245 (2016).

[58] J. E. Smith, B. Mussard, A. A. Holmes, and S. Sharma, J. Chem. Theory Comput. 13, 5468 (2017).

[59] F. Schautz and C. Filippi, J. Chem. Phys. 120, 10931 (2004).

[60] J. Toulouse and C. J. Umrigar, J. Chem. Phys. 126, 084102 (2007).

[61] C. J. Umrigar, J. Toulouse, C. Filippi, S. Sorella, and R. G. Hennig, Phys. Rev. Lett. 98, 110201 (2007).

[62] H. Luo, J. Chem. Phys. 135, 024109 (2011).

[63] K. Haghighi Mood and A. Lüchow, J. Phys. Chem. A 121, 6165 (2017).

[64] J. Ludovicy, K. H. Mood, and A. Lüchow, J. Chem. Theory Comput. 15, 5221 (2019).

[65] N. C. Rubin, R. Babbush, and J. McClean, New J. Phys. 20, 053020 (2018).

[66] A. F. Izmaylov, T.-C. Yen, R. A. Lang, and V. Verteletskyi, J. Chem. Theory Comput. 16, 190 (2019).

[67] V. Verteletskyi, T.-C. Yen, and A. F. Izmaylov, J. Chem. Phys. 152, 124114 (2020).

[68] T.-C. Yen, V. Verteletskyi, and A. F. Izmaylov, J. Chem. Theory Comput. 16, 2400 (2020).

[69] A. F. Izmaylov, T.-C. Yen, and I. G. Ryabinkin, Chem. Sci. 10, 3746 (2019).

[70] A. Jena, S. Genin, and M. Mosca, arXiv:1907.07859.

[71] P. Gokhale, O. Angiuli, Y. Ding, K. Gui, T. Tomesh, M. Suchara, M. Martonosi, and F. T. Chong, arXiv:1907.13623.

[72] W. J. Huggins, J. McClean, N. Rubin, Z. Jiang, N. Wiebe, K. B. Whaley, and R. Babbush, arXiv:1907.13117.

[73] G. Torlai, G. Mazzola, G. Carleo, and A. Mezzacapo, Phys. Rev. Res. 2, 022060 (2020).

[74] A. Zhao, A. Tranter, W. M. Kirby, S. F. Ung, A. Miyake, and P. J. Love, Phys. Rev. A 101, 062322 (2020).

[75] I. Hamamura and T. Imamichi, npj Quantum Inf. 6, 56 (2020).
[76] N. C. Handy and H. F. Schaefer III, J. Chem. Phys. 81, 5031 (1984).

[77] T. Helgaker and P. Jørgensen, Theor. Chim. Acta 75, 111 (1989).

[78] R. M. Parrish, E. G. Hohenstein, P. L. McMahon, and T. J. Martinez, arXiv:1906.08728.

[79] K. Mitarai, Y. O. Nakagawa, and W. Mizukami, Phys. Rev. Res. 2, 013129 (2020).

[80] P. K. Barkoutsos, J. F. Gonthier, I. Sokolov, N. Moll, G. Salis, A. Fuhrer, M. Ganzhorn, D. J. Egger, M. Troyer, A. Mezzacapo, S. Filipp, and I. Tavernelli, Phys. Rev. A 98, 022322 (2018).

[81] H. R. Grimsley, D. Claudino, S. E. Economou, E. Barnes, and N. J. Mayhall, J. Chem. Theory Comput. 16, 1 (2019).

[82] A. F. Izmaylov, M. Díaz-Tinoco, and R. A. Lang, Phys. Chem. Chem. Phys. 22, 12980 (2020).

[83] Q. Sun, T. C. Berkelbach, N. S. Blunt, G. H. Booth, S. Guo, Z. Li, J. Liu, J. D. McClain, E. R. Sayfutyarova, S. Sharma, S. Wouters, and G. K. Chan, Wiley Interdiscip. Rev.: Comput. Mol. Sci. 8, e1340 (2017).

[84] J. R. McClean, K. J. Sung, I. D. Kivlichan, Y. Cao, C. Dai, E. S. Fried, C. Gidney, B. Gimby, P. Gokhale, T. Häner, T. Hardikar, V. Havlíçek, O. Higgott, C. Huang, J. Izaac, Z. Jiang, X. Liu, S. McArdle, M. Neeley, T. O’Brien, B. O'Gorman, I. Ozfidan, M. D. Radin, J. Romero, N. Rubin, N. P. D. Sawaya, K. Setia, S. Sim, D. S. Steiger, M. Steudtner, Q. Sun, W. Sun, D. Wang, F. Zhang, and R. Babbush, Quantum Science and Technology 5, 034014 (2020).

[85] Q. Sun, J. Yang, and G. K.-L. Chan, Chem. Phys. Lett. 683, 291 (2017).

[86] F. Neese, Wiley Interdiscip. Rev.: Comput. Mol. Sci. 2, 73 (2012).

[87] F. Neese, Wiley Interdiscip. Rev.: Comput. Mol. Sci. 8, e1327 (2018).

[88] J. Preskill, Quantum 2, 79 (2018).

[89] F. Arute, K. Arya, R. Babbush, D. Bacon, J. C. Bardin, R. Barends, R. Biswas, S. Boixo, F. G. S. L. Brandao, D. A. Buell, B. Burkett, Y. Chen, Z. Chen, B. Chiaro, R. Collins, W. Courtney, A. Dunsworth, E. Farhi, B. Foxen, A. Fowler, C. Gidney, M. Giustina, R. Graff, K. Guerin, S. Habegger, M. P. Harrigan, M. J. Hartmann, A. Ho, M. Hoffmann, T. Huang, T. S. Humble, S. V. Isakov, E. Jeffrey, Z. Jiang, D. Kafri, K. Kechedzhi, J. Kelly, P. V. Klimov, S. Knysh, A. Korotkov, F. Kostritsa, D. Landhuis, M. Lindmark, E. Lucero, D. Lyakh, S. Mandrà, J. R. McClean, M. McEwen, A. Megrant, X. Mi, K. Michielsen, M. Mohseni, J. Mutus, O. Naaman, M. Neeley, C. Neill, M. Y. Niu, E. Ostby, A. Petukhov, J. C. Platt, C. Quintana, E. G. Rieffel, P. Roushan, N. C. Rubin, D. Sank, K. J. Satzinger, V. Smelyanskiy, K. J. Sung, M. D. Trevithick, A. Vainsencher, B. Villalonga, T. White, Z. J. Yao, P. Yeh, A. Zalcman, H. Neven, and J. M. Martinis, Nature (London) 574, 505 (2019). 